\title{
Indexing of Under Groundwater Quality in Urban Area of Moradabad District in Northern India
}

\author{
Rajeev KumarSingh ${ }^{1}$,Ritu Singh $^{2}$ \\ ${ }^{I}$ Shri Ram MuratiSamarak College of engineering and technology, Bareilly (up) \\ ${ }^{2}$ Shri Ram MuratiSamarak College of engineering, technology \& Research Bareilly (up)
}

\begin{abstract}
A study was carried out to assess the ground water quality of urban area of Moradabad, a developing industrial city of Uttar Pradesh. The water samples were collected from bore wells, tube wells and hand pumps in research area. Various physico-chemical parameters were analysed. The $p H$ value was in proximity to benchmarked NPI index value i.e. 1.0, while the NPI value for total alkalinity was found to be above one. The NPI values for calcium, magnesium, phosphate, chlorides, total dissolved solids, total hardness and nitrate were far below the critical NPI value of 1.00
\end{abstract}

Key Word: Ground water, Moradabad, urban area, water quality

\section{Introduction}

The quality of water is depleting rapidly with the change in human life style i.e.,Massive industrialization, construction activities, utilization of agricultural land and forestLand for other developmental purposes. Most of the groundwater studies focus on theAssessment of quantitative sources and on the identification recharge processes. TheseDevelopmental activities generate large quantities of concentrated effluents. These effluentsare either dumped into ground or drained into open unlined earthen canals from where theyPercolate, resulting groundwater pollution.Moradabad is a developing industrial city situated at a distance of $167 \mathrm{~km}$ from the national capital, New Delhi (NH 24), on the bank of river Ram Ganga and is located between $28021^{\prime}$ 'to $280-16^{\prime}$ ' latitude north and 780 4' longitude east. It has an average elevation of 186 meters (610 feet) above sea level. The drinking water is available in the form of municipal taps, hand pumps ,jet pumps, bore wells etc. but most of the population depends upon the underground water both for domestic and irrigation purposes .undoubtedly the infested pollution load caused by rapid urbanization \& industrialization is harming water quality so as to establish a better water management plan and to monitor water quality, it is very important to know different physio chemical parameters of water, namely colour , odour ,taste , $\mathrm{pH}$,electrical conductance (EC), total dissolved solids (TDS), turbidity ,total hardness (TH), total alkalinity (TA) , chloride (Cl-) , nitrate (NO3-) ,phosphate (PO4)-- ,sulphate(SO4)--,heavy metal. Water quality index (WQI) was calculated which reflects the collective criteria of the drinking water quality of the urban area of the city.

\section{Methodology}

The samples were collected in triplicates from the sites marked 1 to 10 of experimental zone in three different seasons i.e. summer, monsoon and winters of the year 2014-2015. They were preserved, marked and analysed according to standard methods (APHA, 1992). The $\mathrm{pH}$ was measured using $\mathrm{pH}$ meter (SYSTRONICS 335) while total dissolved solids and turbidity were measured by conductivity meter and turbidity meter (ELICO CL-52) respectively Chloride was measured argentometrically whereas sulphate, nitrate and phosphates were determined spectrophotometrically, while total hardness, total alkalinity, calcium and magnesium were measured titrimetricaly. Analysis of heavy metals in water was done by atomic absorption spectrophotometer Perkin Elmer Model 130 .The water quality was assessed using Nemerow's pollution index (NPI), mathematically expressed as

$\mathrm{NPI}=\mathrm{Ci} / \mathrm{Li}$

Where $\mathrm{Ci}$ and $\mathrm{Li}$ are observed concentration and permissible limit of $\mathrm{i}^{\text {th }}$ parameter respectively. The NPI value exceeding 1.00 indicates the presence of pollutant parameter in water sample.

\section{Result And Discussion}

All parameters were measured in $\mathrm{mg} / \mathrm{L}$ except hardness which measured as $\mathrm{CaCO} 3$. Analytical results were compiled and shown in Table 1 and various sample point locations are shown in Table 2.Observed changes in various parameters are discussed below.The $\mathrm{pH}$ ranged from 7.55 to 8.03 showing alkaline nature of ground water which was probably due to presence of carbonates and bi carbonates. The highest value was at site 4 and lowest at site 6 .The values of PH were found well within the limits of BIS standards. Similar results were shown by Reddy et.al (1999). TDS denote mainly the various kinds of minerals present in water. A higher content of TDS elevates the density of water. The value of TDS is maximum at site $9(488 \mathrm{mg} / \mathrm{L})$ and minimum at site $8(248 \mathrm{mg} / \mathrm{L})$. TDS beyond $500 \mathrm{mg} / \mathrm{L}$. decreases palatability and also favour gastro intestinal diseases. 
TDS value up to $1000 \mathrm{mg} / \mathrm{L}$ is considered Safe for potable purpose according to WHO (1993).Turbidity refers to the relative clarity of water its maximum value at site $4(1.166 \mathrm{NTU})$ and minimum value at site 7 (0.600NTU).Turbidity in water acceptable when it used for domestic washing and bathing. Turbidity was found higher in rainy season in compare to other seasons. The NPI value of $\mathrm{pH}$ was just below standard index bench mark 1.00, while TDS and Turbidity NPI value are well within the preset standard.As prescribed limit of chloride by WHO are $250 \mathrm{mg} / \mathrm{L}$. all samples were within the permissible range. It is found maximum at site 9 (86.13) and minimum at site 4 (36.1). Even Sulphate, which imparts hardness to the water, was maximum at site 9 (58.33) and minimum at site 4, 5 (20). The concentration of phosphate was very less In sub surface water with maximum at site 1,3,10 (0.0166) and minimum at site 7 (0066). No regular pattern of seasonal variation was observed the water containing more than $50 \mathrm{mg} / \mathrm{L} \mathrm{NO}-\mathrm{N}$ is unsafe for drinking purposes as recommended by WHO the lowest value was at site $8(0.0133)$ and highest at site 9 (0.097). The NPI value calculated for chloride, sulphate, nitrate and phosphate were again found to be well within the critical and alarming presumed index indicator 1.00.Total alkalinity is a measure of an aggregate property of water. The maximum value was at site $1(280 \mathrm{mg} / \mathrm{L})$ and lowest at site $7(200 \mathrm{mg} / \mathrm{L})$. The NPI of alkalinity was found to be greater than 1.00 for all the samples. It is found that high alkalinity imparts bitter taste to water making it unpalatable. Hardness of water is mainly due to calcium and magnesium present in it. The maximum and minimum value of total hardness was observed at site $2(274 \mathrm{mg} / \mathrm{L})$ and site $9(162 \mathrm{mg} / \mathrm{L})$ respectively. The calcium content varied from 29.51 (site 5) to $74.16 \mathrm{mg} / \mathrm{L}$ (site8) and the magnesium concentration ranged from $58.39 \mathrm{mg} / \mathrm{L}$ (site 8 ) to $133.15 \mathrm{mg} / \mathrm{L}$ (site 4 ). In low concentration these are nontoxic but in high concentration are not desirable washing, laundering $\&$ cooking purposes (singh et al 1999). The permissible range for heavy metals like $\mathrm{Fe}, \mathrm{Pb}, \mathrm{Zn}, \mathrm{Cr}, \mathrm{Ni}, \mathrm{Cu}$ are 0.3 $\mathrm{mg} / \mathrm{L}, 0.05 \mathrm{mg} / \mathrm{L}, 5 \mathrm{mg} / \mathrm{L}, 0.05 \mathrm{mg} / \mathrm{L}, 0.05 \mathrm{mg} / \mathrm{L}, 0.05 \mathrm{mg} / \mathrm{L}$ respectively .Most of the samples showed Higher Value of iron content in ground water. Zinc and Chromium concentration exceeded the prescribed limit whereas Copper and nickel concentration were well within the prescribed limit for most of the samples. However, no regular trend was seen in the three seasons

Table 1: Physico-Chemical parameters and NPI of ten sites in densely populated area of Moradabad city.

\begin{tabular}{|c|c|c|c|c|c|c|c|c|c|c|c|c|c|c|c|c|c|c|c|}
\hline \multicolumn{7}{|c|}{ MIandisamiti Sate-1 } & \multicolumn{6}{|c|}{ Locoskedbridge Site -2} & \multicolumn{6}{|c|}{ MIDA Site -3} & \multirow{2}{*}{$\begin{array}{l}\text { WHO } \\
\text { CPHEE } \\
\text { o } \\
\text { Send (ms } \\
\text { L) }\end{array}$} \\
\hline $\begin{array}{l}\text { Paramat } \\
\text { ex }\end{array}$ & $\begin{array}{l}\text { Sum } \\
\text { mer }\end{array}$ & $\begin{array}{l}\mathrm{Rz} \\
\mathrm{my}\end{array}$ & $\begin{array}{l}\text { Wis } \\
\text { ex }\end{array}$ & $\begin{array}{l}\text { Avera } \\
\text { De }\end{array}$ & $S D=$ & NPI & $\begin{array}{l}\text { Ssim } \\
\text { met }\end{array}$ & $\begin{array}{l}\text { Pui } \\
\text { ny }\end{array}$ & $\begin{array}{l}\text { Wint } \\
\text { et }\end{array}$ & $\begin{array}{l}\text { Avera } \\
\text { ge }\end{array}$ & $S D=$ & NPI & $\begin{array}{l}\text { Sumt } \\
\text { mer }\end{array}$ & $\begin{array}{l}\text { Rui } \\
\text { ny }\end{array}$ & $\begin{array}{l}\text { Wint } \\
\text { et }\end{array}$ & $\begin{array}{l}\text { Avera } \\
\text { de }\end{array}$ & $S \mathrm{D}=$ & NPI & \\
\hline $\mathrm{pH}$ & 7.21 & $\begin{array}{l}8.2 \\
7\end{array}$ & 7.51 & 7.66 & $3^{0.546}$ & 0.9 & 702 & $\begin{array}{l}3.1 \\
5\end{array}$ & 7.67 & 7.61 & $\begin{array}{l}0.567 \\
1273\end{array}$ & $\begin{array}{l}0.89 \\
5\end{array}$ & 7.29 & $\begin{array}{l}8.5 \\
3.5\end{array}$ & 7.23 & 7.69 & $\begin{array}{l}0.745 \\
3858\end{array}$ & 69 & $8.5-6.5$ \\
\hline TDS & 273.3 & $\begin{array}{l}313 \\
3\end{array}$ & $\begin{array}{l}313 . \\
3\end{array}$ & 299.9 & $\begin{array}{l}25.04 \\
01\end{array}$ & $\begin{array}{l}0.29 \\
9\end{array}$ & 400 & $\begin{array}{c}413 \\
3\end{array}$ & $\begin{array}{l}246 . \\
6\end{array}$ & 355.3 & $\begin{array}{l}92.54 \\
3858\end{array}$ & $\begin{array}{l}0.35 \\
3\end{array}$ & 3406 & 400 & $\begin{array}{c}3500 \\
6\end{array}$ & $\frac{3510}{7}$ & $\begin{array}{l}1693 \\
752\end{array}$ & $\begin{array}{l}0.35 \\
1\end{array}$ & 1000 \\
\hline $\begin{array}{l}\text { Turbid } \\
\text { ity }\end{array}$ & 0.2 & 1.5 & 0.4 & 0.7 & 0.7 & 0.14 & 0.5 & 1.7 & 0.4 & 0.87 & $\begin{array}{l}0.723 \\
4178\end{array}$ & $\begin{array}{l}0.17 \\
4 \\
\end{array}$ & 0.2 & 2 & 40.1 & 0.77 & $\begin{array}{l}22.53 \\
464\end{array}$ & $\begin{array}{l}0.15 \\
4\end{array}$ & S.NTU \\
\hline $\mathrm{Cl}^{-}$ & 73.8 & $\begin{array}{l}+2 . \\
6\end{array}$ & 625 & 3965 & $\begin{array}{l}15.79 \\
6308\end{array}$ & $\begin{array}{l}0.23 \\
9 \\
9\end{array}$ & $n$ & $\begin{array}{l}36 . \\
9\end{array}$ & 02.5 & 36.8 & $\begin{array}{l}17.75 \\
0211\end{array}$ & $\begin{array}{l}0.22 \\
7\end{array}$ & 79.5 & $\begin{array}{l}31 . \\
2\end{array}$ & 42.6 & 31.1 & $\begin{array}{l}25.24 \\
698\end{array}$ & $\begin{array}{l}0.20 \\
4 \\
\end{array}$ & 250 \\
\hline $\mathrm{SO}_{4}$ & 40 & 15 & 95 & 30 & $\begin{array}{l}40.92 \\
6764\end{array}$ & $\begin{array}{l}0.12 \\
5\end{array}$ & 50 & 40 & 65 & 31.66 & $\begin{array}{l}1238 \\
3057\end{array}$ & $\begin{array}{l}0.12 \\
9\end{array}$ & 30 & 15 & 10 & 18,33 & $\begin{array}{l}10.40 \\
833\end{array}$ & $\begin{array}{l}0.04 \\
6\end{array}$ & 400 \\
\hline TA & 240 & 460 & 140 & 280 & $\begin{array}{l}163.7 \\
0700\end{array}$ & ${ }_{3}^{233}$ & 260 & 420 & 140 & \begin{tabular}{|l|}
273.3 \\
3
\end{tabular} & $\begin{array}{l}140.4 \\
7538\end{array}$ & 2.28 & 2200 & 300 & 120 & $\begin{array}{l}2133 \\
3\end{array}$ & $\begin{array}{l}9018 \\
4995\end{array}$ & 1.78 & 120 \\
\hline $\mathrm{TH}$ & 185 & 250 & 280 & $\begin{array}{l}2395 \\
3\end{array}$ & $\begin{array}{l}46.91 \\
8369\end{array}$ & $\begin{array}{l}0.47 \\
8\end{array}$ & 158 & 350 & 284 & 274 & $\begin{array}{l}81.46 \\
1647\end{array}$ & $\begin{array}{l}0.54 \\
8\end{array}$ & 90 & 240 & 320 & $\begin{array}{l}2186 \\
7\end{array}$ & $\begin{array}{l}113.5 \\
1358\end{array}$ & $\begin{array}{l}0.43 \\
7\end{array}$ & 500 \\
\hline $\mathrm{NO}_{4}$ & 0.06 & $\begin{array}{l}0.0 \\
4\end{array}$ & 0.03 & 0.043 & $\begin{array}{l}0.015 \\
2753\end{array}$ & $\begin{array}{l}0.00 \\
028\end{array}$ & 01 & $\begin{array}{l}0.0 \\
2\end{array}$ & 0.05 & 0.057 & $\begin{array}{l}0.040 \\
4 ! 45\end{array}$ & $\begin{array}{l}0.00 \\
038\end{array}$ & 0.19 & $2^{0.0}$ & 0.08 & 0.097 & $\begin{array}{l}0.086 \\
2168\end{array}$ & $\begin{array}{l}0.00 \\
065\end{array}$ & 50 \\
\hline $\mathrm{PO}_{4}$ & 0.01 & $\begin{array}{l}0.0 \\
2\end{array}$ & 0.02 & $\begin{array}{l}0.015 \\
6\end{array}$ & $\begin{array}{l}0.005 \\
7735\end{array}$ & $\begin{array}{l}0.16 \\
6\end{array}$ & 0.01 & 0 & 0.03 & $\begin{array}{l}0.013 \\
3\end{array}$ & $\begin{array}{l}0.015 \\
2753\end{array}$ & $\begin{array}{l}0.119 \\
3\end{array}$ & 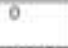 & $\begin{array}{l}800 \\
2\end{array}$ & 0.03 & $\begin{array}{l}0.016 \\
6\end{array}$ & $\begin{array}{l}0015 \\
2753\end{array}$ & $\begin{array}{l}0.16 \\
6\end{array}$ & 0.1 \\
\hline $\mathrm{M} / \mathrm{s}$ & 36.03 & $\begin{array}{l}74 . \\
96 \\
\end{array}$ & $\begin{array}{l}184 . \\
1\end{array}$ & 9830 & $\begin{array}{l}70.75 \\
9157\end{array}$ & $\begin{array}{l}0.65 \\
6 \\
\end{array}$ & 35.27 & $\begin{array}{l}110 \\
25\end{array}$ & $\begin{array}{l}101 . \\
4\end{array}$ & $\begin{array}{l}102.3 \\
1\end{array}$ & $\begin{array}{l}63.43 \\
9078\end{array}$ & $\begin{array}{l}0.68 \\
2\end{array}$ & 45.08 & $\begin{array}{l}76 . \\
25\end{array}$ & $\begin{array}{l}219 . \\
1\end{array}$ & $\begin{array}{l}113.4 \\
8\end{array}$ & $\begin{array}{l}92.79 \\
0671\end{array}$ & $\begin{array}{l}0.07 \\
65\end{array}$ & 150 \\
\hline a & 4469 & $\begin{array}{l}52 \\
9\end{array}$ & $\begin{array}{l}45.4 \\
0\end{array}$ & 47.68 & $\begin{array}{l}534 \\
1409\end{array}$ & $\begin{array}{l}0.23 \\
8\end{array}$ & 76.28 & $\begin{array}{l}68 \\
95\end{array}$ & $\begin{array}{l}60.0 \\
8\end{array}$ & 68.43 & $\begin{array}{l}8.111 \\
5658\end{array}$ & $\begin{array}{l}0.34 \\
2\end{array}$ & 22,4 & 33 & $\begin{array}{l}39.6 \\
1\end{array}$ & 31.89 & $\begin{array}{l}8.740 \\
4634\end{array}$ & $\begin{array}{l}0.15 \\
90 \mathrm{~V}\end{array}$ & 200 \\
\hline
\end{tabular}

Cont............

\begin{tabular}{|c|c|c|c|c|c|c|c|c|c|c|c|c|c|c|c|c|c|c|c|}
\hline \multicolumn{7}{|c|}{ Cwill Limes Site -4} & \multicolumn{6}{|c|}{ Mloradabad Club Site-5 } & \multicolumn{6}{|c|}{ Harthala Colony site -6} & \multirow{2}{*}{$\begin{array}{l}\text { WHO } \\
\text { CPHE } \\
\text { EO } \\
\text { Strd. } \\
\text { meL.) }\end{array}$} \\
\hline $\begin{array}{l}\text { Paramet } \\
\text { ex }\end{array}$ & $\begin{array}{l}\text { Sum } \\
\text { mer }\end{array}$ & $\begin{array}{l}\text { Rai } \\
\text { ny }\end{array}$ & $\begin{array}{l}\text { Wint } \\
\text { et }\end{array}$ & $\begin{array}{l}\text { Avera } \\
\text { ge }\end{array}$ & $S D=$ & NPI & $\begin{array}{l}\text { Sum } \\
\text { mer }\end{array}$ & $\begin{array}{l}\text { Rai } \\
\text { ay }\end{array}$ & $\begin{array}{l}\text { Wint } \\
\text { et }\end{array}$ & $\begin{array}{l}\text { Avera } \\
\text { ge }\end{array}$ & $S D=$ & NPI & $\begin{array}{l}\text { Sam } \\
\text { mer }\end{array}$ & $\begin{array}{l}\text { Rail } \\
\text { ay }\end{array}$ & $\begin{array}{l}\text { Wint } \\
\text { et }\end{array}$ & $\begin{array}{l}\text { Avera } \\
\text { ge }\end{array}$ & $5 D=$ & NP1 & \\
\hline $\mathrm{pH}$ & 7.4 & $\begin{array}{l}8.8 \\
9\end{array}$ & 7.81 & 803 & $\begin{array}{l}0.769 \\
6969\end{array}$ & 0.94 & 7.44 & $\begin{array}{l}8.7 \\
9 \\
\end{array}$ & 7.48 & 7.9 & $\begin{array}{l}0.768 \\
1363\end{array}$ & $\begin{array}{l}0.9 \\
3\end{array}$ & 736 & $\begin{array}{l}8.4 \\
7\end{array}$ & 6.83 & 7.55 & $\begin{array}{l}0.836 \\
919\end{array}$ & $\begin{array}{l}0.8 \\
88\end{array}$ & 8.5 .65 \\
\hline TDS & 200 & $\begin{array}{l}333 \\
3\end{array}$ & $\begin{array}{l}306 . \\
6\end{array}$ & $\begin{array}{l}2799 \\
7\end{array}$ & $\begin{array}{l}70.52 \\
81744\end{array}$ & $\begin{array}{l}0.27 \\
9\end{array}$ & 306.6 & 400 & $\begin{array}{l}253 \\
3\end{array}$ & $\begin{array}{l}319.9 \\
6\end{array}$ & $\begin{array}{l}74.25 \\
7817\end{array}$ & $\begin{array}{l}0.3 \\
19\end{array}$ & 300 & $\begin{array}{c}366 \\
6\end{array}$ & $\begin{array}{l}253 \\
3\end{array}$ & $\begin{array}{l}306.6 \\
3\end{array}$ & $\begin{array}{l}5694 \\
052\end{array}$ & $\begin{array}{l}0.3 \\
06\end{array}$ & 1000 \\
\hline $\begin{array}{l}\text { Turbi } \\
\text { Eity }\end{array}$ & 0.1 & 1.8 & 0.1 & 0.6 & $\begin{array}{l}0.806 \\
0254\end{array}$ & 0.12 & 0.4 & 2 & 0.2 & 0.87 & $\begin{array}{l}0,956 \\
5766\end{array}$ & $\begin{array}{l}0.1 \\
74\end{array}$ & 02 & 1.9 & 1 & 1033 & $\begin{array}{l}0.850 \\
49\end{array}$ & $\begin{array}{l}0.2 \\
07\end{array}$ & $5 \mathrm{NTU}$ \\
\hline$a^{2}$ & 31.6 & $\begin{array}{l}28 \\
4\end{array}$ & 48.3 & 36.1 & $\begin{array}{l}10,68 \\
597\end{array}$ & $\begin{array}{l}0.14 \\
4\end{array}$ & 65.3 & $\begin{array}{l}25 . \\
6\end{array}$ & 39.7 & 43.53 & $\begin{array}{l}20.12 \\
5688\end{array}$ & $\begin{array}{l}0.1 \\
74\end{array}$ & 65.3 & $\begin{array}{l}56 . \\
8\end{array}$ & 455 & 55.87 & $\begin{array}{l}227.4 \\
868\end{array}$ & $\begin{array}{l}0.2 \\
23\end{array}$ & 250 \\
\hline $\mathrm{SO}_{i}$ & 15 & 30 & 15 & 20 & $\begin{array}{l}8.660 \\
254\end{array}$ & 0.05 & 25 & 10 & 25 & 20 & $\begin{array}{l}8.660 \\
254\end{array}$ & $\begin{array}{l}0.0 \\
5 \\
\end{array}$ & 80 & 35 & 45 & 53.33 & $\begin{array}{l}23.62 \\
908\end{array}$ & $\begin{array}{l}0.1 \\
33\end{array}$ & 400 \\
\hline TA & 260 & 240 & 140 & $\begin{array}{l}2133 \\
3\end{array}$ & $\begin{array}{l}6429 \\
1005\end{array}$ & 1.78 & 220 & 320 & 160 & $\begin{array}{l}233.3 \\
3\end{array}$ & $\begin{array}{l}80.82 \\
9038\end{array}$ & $\begin{array}{l}19 \\
4\end{array}$ & 300 & 320 & 160 & 260 & $\begin{array}{l}87.17 \\
7979\end{array}$ & $\begin{array}{l}2.1 \\
66\end{array}$ & 120 \\
\hline TH & 150 & 300 & 324 & 258 & $\begin{array}{l}94.29 \\
7402\end{array}$ & $\frac{0.51}{6}$ & 170 & 240 & 252 & $\begin{array}{l}220.6 \\
6\end{array}$ & $\begin{array}{l}44.28 \\
6943\end{array}$ & $\begin{array}{l}0.4 \\
4\end{array}$ & 160 & 270 & 356 & 262 & $\begin{array}{l}98.24 \\
4593\end{array}$ & $\begin{array}{l}0.5 \\
24\end{array}$ & 500 \\
\hline No, & 0.09 & $\begin{array}{l}0.0 \\
4\end{array}$ & 0.11 & 0.08 & $\begin{array}{l}0.036 \\
0555\end{array}$ & $\begin{array}{l}0.00 \\
053\end{array}$ & 0.08 & $\begin{array}{l}0.0 \\
6\end{array}$ & 0.04 & 0.06 & 0.02 & $\begin{array}{l}0.0 \\
004\end{array}$ & 0.07 & $\begin{array}{l}0.0 \\
7\end{array}$ & 0.04 & 0.06 & $\begin{array}{l}0.017 \\
3205\end{array}$ & $\begin{array}{l}0.0 \\
004\end{array}$ & 50 \\
\hline $\mathrm{PO}_{N}$ & 0 & $\begin{array}{l}0.0 \\
1\end{array}$ & 0.03 & $\begin{array}{l}0.013 \\
3\end{array}$ & $\begin{array}{l}0.015 \\
2753\end{array}$ & $\begin{array}{l}0.13 \\
3\end{array}$ & 0 & $\begin{array}{l}0.0 \\
1\end{array}$ & 0.02 & 0.01 & 0.01 & 0.1 & 0 & $\begin{array}{l}0.0 \\
1\end{array}$ & 0.02 & 0.01 & 0.01 & 0.1 & 0.1 \\
\hline $\mathrm{Mg}$ & 84.05 & $\begin{array}{l}96 \\
28\end{array}$ & $\begin{array}{l}219 \\
12\end{array}$ & $\begin{array}{l}1331 \\
5\end{array}$ & $\begin{array}{l}74.70 \\
2904\end{array}$ & $\begin{array}{l}0.88 \\
8\end{array}$ & 98.85 & $\begin{array}{l}78 \\
24\end{array}$ & $\begin{array}{l}141 \\
87\end{array}$ & $\begin{array}{l}1063 \\
2\end{array}$ & $\begin{array}{l}32.46 \\
6058\end{array}$ & $\begin{array}{l}0.7 \\
09\end{array}$ & 22.44 & $\begin{array}{l}80 \\
02\end{array}$ & $\begin{array}{l}207 \\
9\end{array}$ & $\begin{array}{l}105.4 \\
5\end{array}$ & $\begin{array}{l}9424 \\
4861\end{array}$ & $\begin{array}{l}0.7 \\
03\end{array}$ & 150 \\
\hline $\mathrm{C}_{3}$ & 482 & $\begin{array}{l}36 \\
87\end{array}$ & $\begin{array}{l}40.5 \\
9\end{array}$ & 41.88 & $\begin{array}{l}5.775 \\
2258\end{array}$ & $\begin{array}{l}0.20 \\
9\end{array}$ & 26.48 & $\begin{array}{l}22 \\
44\end{array}$ & $\begin{array}{l}39.6 \\
1\end{array}$ & 29.51 & $\begin{array}{l}8.977 \\
0764\end{array}$ & $\begin{array}{l}0.1 \\
48\end{array}$ & 84.01 & $\begin{array}{l}33 . \\
66\end{array}$ & 56.2 & 57.62 & $\begin{array}{l}25.22 \\
0925\end{array}$ & $\begin{array}{l}0.2 \\
88\end{array}$ & 200 \\
\hline
\end{tabular}


Cont.

\begin{tabular}{|c|c|c|c|c|c|c|c|c|c|c|c|c|c|c|c|c|c|c|c|}
\hline \multicolumn{7}{|c|}{ Mahila Thasua Site -7} & \multicolumn{6}{|c|}{ DyptiGanj Site - 8} & \multicolumn{6}{|c|}{ Jain Mlandir Site - 9} & \multirow{2}{*}{$\begin{array}{l}\text { WHO } \\
\text { CPHE } \\
\text { EO } \\
\text { Stuf( } \\
\text { mg L) }\end{array}$} \\
\hline mameter & $\begin{array}{l}\text { Sum } \\
\text { mer }\end{array}$ & $\begin{array}{l}\text { Rai } \\
\text { ay }\end{array}$ & $\begin{array}{l}\text { Wiut } \\
\text { ef }\end{array}$ & $\begin{array}{l}\text { Avera } \\
\text { ge }\end{array}$ & $\mathrm{SD}=$ & NPI & $\begin{array}{l}\text { Suli } \\
\text { met }\end{array}$ & $\begin{array}{l}\text { Rai } \\
\text { ny }\end{array}$ & $\begin{array}{l}\text { Wint } \\
\text { ef }\end{array}$ & $\begin{array}{l}\text { Avera } \\
\text { ge }\end{array}$ & $S D=$ & NPI & $\begin{array}{l}\text { Sum } \\
\text { mer }\end{array}$ & $\begin{array}{l}\text { Rai } \\
\text { ny }\end{array}$ & $\begin{array}{l}\text { Whnt } \\
\text { ef }\end{array}$ & $\begin{array}{l}\text { Aven } \\
\text { ge }\end{array}$ & $5 \mathrm{D}=$ & NPI & \\
\hline $\mathrm{pH}$ & 7.39 & $\begin{array}{l}8.6 \\
4\end{array}$ & 7.16 & 7.73 & $\begin{array}{l}0.796 \\
4295\end{array}$ & $\begin{array}{l}0.9 \\
09\end{array}$ & 757 & $\begin{array}{l}8.7 \\
2\end{array}$ & 73 & 786 & $\begin{array}{l}0.754 \\
0778\end{array}$ & $\begin{array}{l}0.9 \\
25\end{array}$ & 7.54 & $\begin{array}{l}8.0 \\
4\end{array}$ & 737 & 7.82 & $\begin{array}{l}0.659 \\
42\end{array}$ & 0.92 & $\begin{array}{l}8.5- \\
6.5\end{array}$ \\
\hline IDS & 4066 & $\begin{array}{c}466 \\
6\end{array}$ & $\begin{array}{l}393 . \\
3\end{array}$ & $\begin{array}{l}4221 \\
?\end{array}$ & $\begin{array}{l}39.05 \\
0779\end{array}$ & $\begin{array}{l}0.4 \\
22\end{array}$ & 220 & 320 & $\begin{array}{l}206 . \\
6\end{array}$ & ${ }_{7}^{248.8}$ & $\begin{array}{l}61.96 \\
655\end{array}$ & $\begin{array}{l}0.2 \\
48\end{array}$ & 500 & $\begin{array}{c}466 \\
6\end{array}$ & 500 & $\begin{array}{l}488.8 \\
6\end{array}$ & $\begin{array}{l}19.28 \\
3499\end{array}$ & $\begin{array}{l}0.48 \\
5\end{array}$ & 1000 \\
\hline $\begin{array}{l}\text { Turbi } \\
\text { fity }\end{array}$ & 1 & 2.1 & 0.4 & 1106 & $\begin{array}{l}0.802 \\
1678\end{array}$ & $\begin{array}{l}0.2 \\
33\end{array}$ & 0.1 & 0.9 & 105 & 0.833 & $\begin{array}{r}60.33 \\
4429 \\
\end{array}$ & $\begin{array}{l}0.1 \\
666\end{array}$ & 0.4 & $t$ & 15 & 0.97 & $\begin{array}{l}0.550 \\
7571\end{array}$ & $\begin{array}{l}0.19 \\
4\end{array}$ & $\begin{array}{l}5 \\
\text { NTU }\end{array}$ \\
\hline $\mathrm{c}^{2}$ & 62.4 & $\begin{array}{l}39 \\
7\end{array}$ & 596 & 53.9 & $\begin{array}{l}1237 \\
6995\end{array}$ & $\begin{array}{l}02 \\
16\end{array}$ & 42.6 & $\begin{array}{l}99 \\
4 \\
\end{array}$ & 39.7 & 60.56 & $\begin{array}{l}33.66 \\
1897\end{array}$ & $\begin{array}{l}0.2 \\
42\end{array}$ & 122.1 & $\begin{array}{l}56 . \\
8\end{array}$ & 79.5 & 86.13 & $\begin{array}{l}33.15 \\
1521\end{array}$ & $\begin{array}{l}0.34 \\
4\end{array}$ & 250 \\
\hline $\mathrm{sol}^{-}$ & 30 & 25 & 25 & 26.66 & $\begin{array}{l}2.886 \\
7513\end{array}$ & 0.0 & 20 & 66 & 45 & 43.66 & $\begin{array}{l}23.02 \\
8967\end{array}$ & $\begin{array}{l}01 \\
09\end{array}$ & 60 & 25 & 90 & 58.33 & $\begin{array}{l}32.53 \\
2035\end{array}$ & $\begin{array}{l}0.14 \\
6\end{array}$ & 400 \\
\hline TA & 200 & 240 & 160 & 200 & 40 & $\begin{array}{l}16 \\
66\end{array}$ & 260 & 340 & 120 & 240 & $\begin{array}{l}111.3 \\
5529\end{array}$ & 2 & 260 & 360 & 140 & $\begin{array}{l}253,3 \\
3\end{array}$ & $\begin{array}{l}110.1 \\
5141\end{array}$ & 2.11 & 120 \\
\hline TH & 164 & 270 & 360 & $\begin{array}{l}264.6 \\
6\end{array}$ & $\begin{array}{l}98.10 \\
8783\end{array}$ & $\begin{array}{l}0.5 \\
29\end{array}$ & 140 & 185 & 204 & $3^{176.3}$ & $\begin{array}{l}32.86 \\
8425\end{array}$ & $\begin{array}{l}0.3 \\
53\end{array}$ & 112 & 150 & 224 & 162 & $\begin{array}{l}56.95 \\
6123\end{array}$ & $\begin{array}{l}0.32 \\
4\end{array}$ & 500 \\
\hline No, & 0.2 & $\begin{array}{l}0.0 \\
4\end{array}$ & 0.08 & 0.046 & $\begin{array}{l}0.083 \\
2666\end{array}$ & $\begin{array}{l}00 \\
031\end{array}$ & 0.04 & 0 & 0 & $\begin{array}{l}0.013 \\
3\end{array}$ & $\begin{array}{l}0.023 \\
094\end{array}$ & $\begin{array}{l}8.6 \\
\text { E. } \\
05\end{array}$ & 0.05 & $\begin{array}{l}0.0 \\
4\end{array}$ & 0.1 & 0.063 & $\begin{array}{l}0.032 \\
1455\end{array}$ & $\begin{array}{l}0.00 \\
042\end{array}$ & 50 \\
\hline $\mathrm{PO}_{4}^{-1}$ & 0 & 0 & 0.02 & $\begin{array}{l}0.006 \\
6\end{array}$ & $\begin{array}{l}0.011 \\
547\end{array}$ & $\begin{array}{l}0.0 \\
06\end{array}$ & 0 & $\begin{array}{l}0.0 \\
1\end{array}$ & 0.02 & 0.01 & 0.01 & 0.1 & 0.01 & 0 & 0.02 & 0.01 & $0.0 \mathrm{t}$ & 0.1 & 0.1 \\
\hline $\mathrm{M} / \mathrm{Z}$ & 39.23 & $\begin{array}{l}98 \\
42 \\
\end{array}$ & $\begin{array}{l}224 \\
3\end{array}$ & $\begin{array}{l}120.6 \\
5\end{array}$ & $\begin{array}{l}94.51 \\
6432\end{array}$ & $\begin{array}{l}0.8 \\
04\end{array}$ & 41.22 & $\begin{array}{l}54 \\
28 \\
\end{array}$ & $\begin{array}{l}79.6 \\
9\end{array}$ & 53.39 & $\begin{array}{l}19.50 \\
2603\end{array}$ & $\begin{array}{l}0.3 \\
89\end{array}$ & 30.84 & $\begin{array}{l}70 . \\
86 \\
\end{array}$ & $\begin{array}{l}84.1 \\
2\end{array}$ & 6194 & $\begin{array}{l}27,73 \\
7419 \\
\end{array}$ & $\begin{array}{l}0.41 \\
3 \\
\end{array}$ & 150 \\
\hline$C_{a}$ & 56.82 & $\begin{array}{l}57 \\
71\end{array}$ & $\begin{array}{l}52.2 \\
8\end{array}$ & 55.6 & $\begin{array}{l}2912 \\
29\end{array}$ & $\begin{array}{l}0.2 \\
78\end{array}$ & 30.48 & $\begin{array}{l}28 \\
85\end{array}$ & $\begin{array}{l}44,4 \\
9\end{array}$ & 74.10 & $\begin{array}{l}8.597 \\
932\end{array}$ & $\begin{array}{l}0.3 \\
71\end{array}$ & 40.26 & $\begin{array}{l}32 \\
06\end{array}$ & $\begin{array}{l}40,4 \\
6\end{array}$ & 37.59 & $\begin{array}{l}4793 \\
0505\end{array}$ & $\begin{array}{l}0.18 \\
8\end{array}$ & 200 \\
\hline
\end{tabular}

Cont............

\begin{tabular}{|c|c|c|c|c|c|c|c|}
\hline \multicolumn{7}{|c|}{ Harpal Nagar Site -10 } & \multirow{2}{*}{$\begin{array}{l}\text { WHO/CPHEE } \\
\text { O Strd.(mg/L) }\end{array}$} \\
\hline Parameter & Summer & Rainy & Winter & Average & SD \pm & NPI & \\
\hline pH & 7.52 & 8.77 & 7.5 & 7.93 & 0.72753 & 0.93 & $8.5-6.5$ \\
\hline TDS & 340 & 473.3 & 446.6 & 419.96 & 70.52817 & 0.419 & 1000 \\
\hline Turbidity & 0.2 & 1.2 & 1.5 & 0.97 & 0.680686 & 0.194 & $5 \mathrm{NTU}$ \\
\hline $\mathrm{Cl}^{-}$ & 116.4 & 19.8 & 88 & 74.73 & 49.64769 & 0.299 & 250 \\
\hline $\mathrm{SO}_{4}^{--}$ & 80 & 5 & 55 & 46.66 & 38.18813 & 0.117 & 400 \\
\hline TA & 240 & 240 & 160 & 213.33 & 46.18802 & 1.78 & 120 \\
\hline TH & 168 & 250 & 216 & 211.33 & 41.19871 & 0.423 & 500 \\
\hline $\mathrm{NO}_{3}^{--}$ & 0.01 & 0.07 & 0.09 & 0.057 & 0.041633 & 0.00038 & 50 \\
\hline $\mathrm{PO}_{4}{ }^{--}$ & 0.01 & 0.01 & 0.03 & 0.0166 & 0.011547 & 0.166 & 0.1 \\
\hline Mg & 25.84 & 110.3 & 82.5 & 72.87 & 43.03525 & 0.486 & 150 \\
\hline $\mathbf{C a}$ & 50.28 & 24.04 & 34.61 & 36.31 & 13.20234 & 0.182 & 200 \\
\hline
\end{tabular}
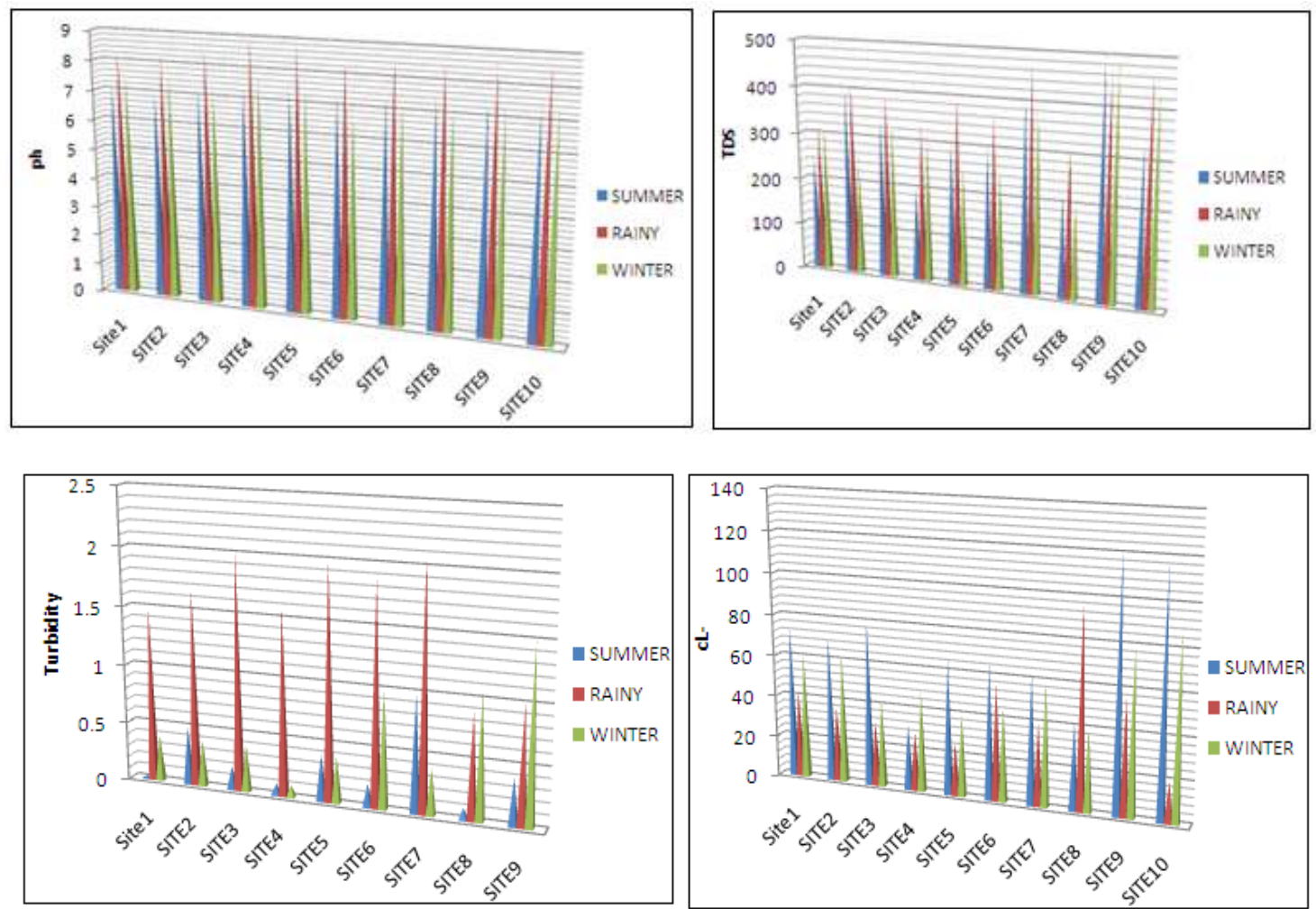

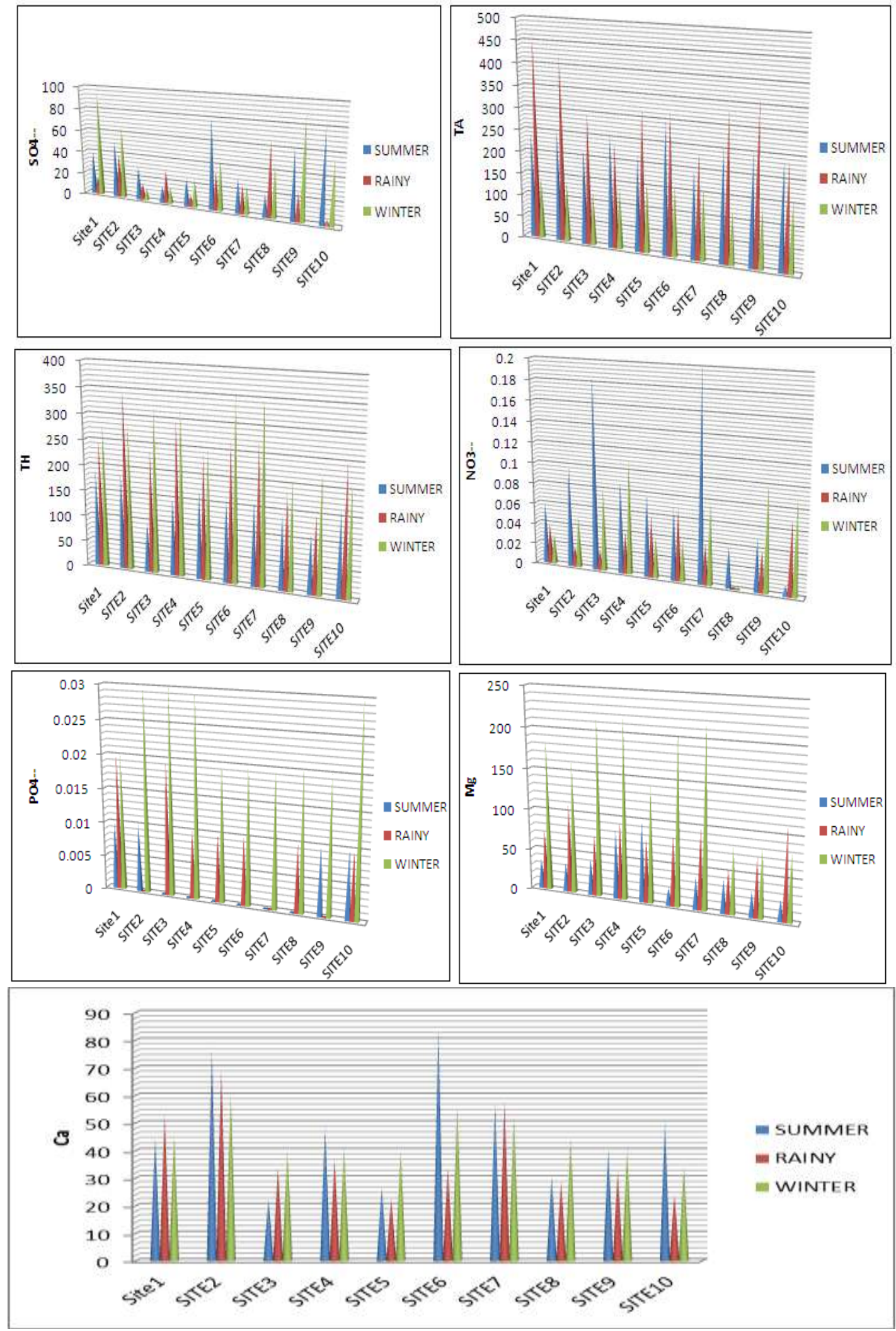


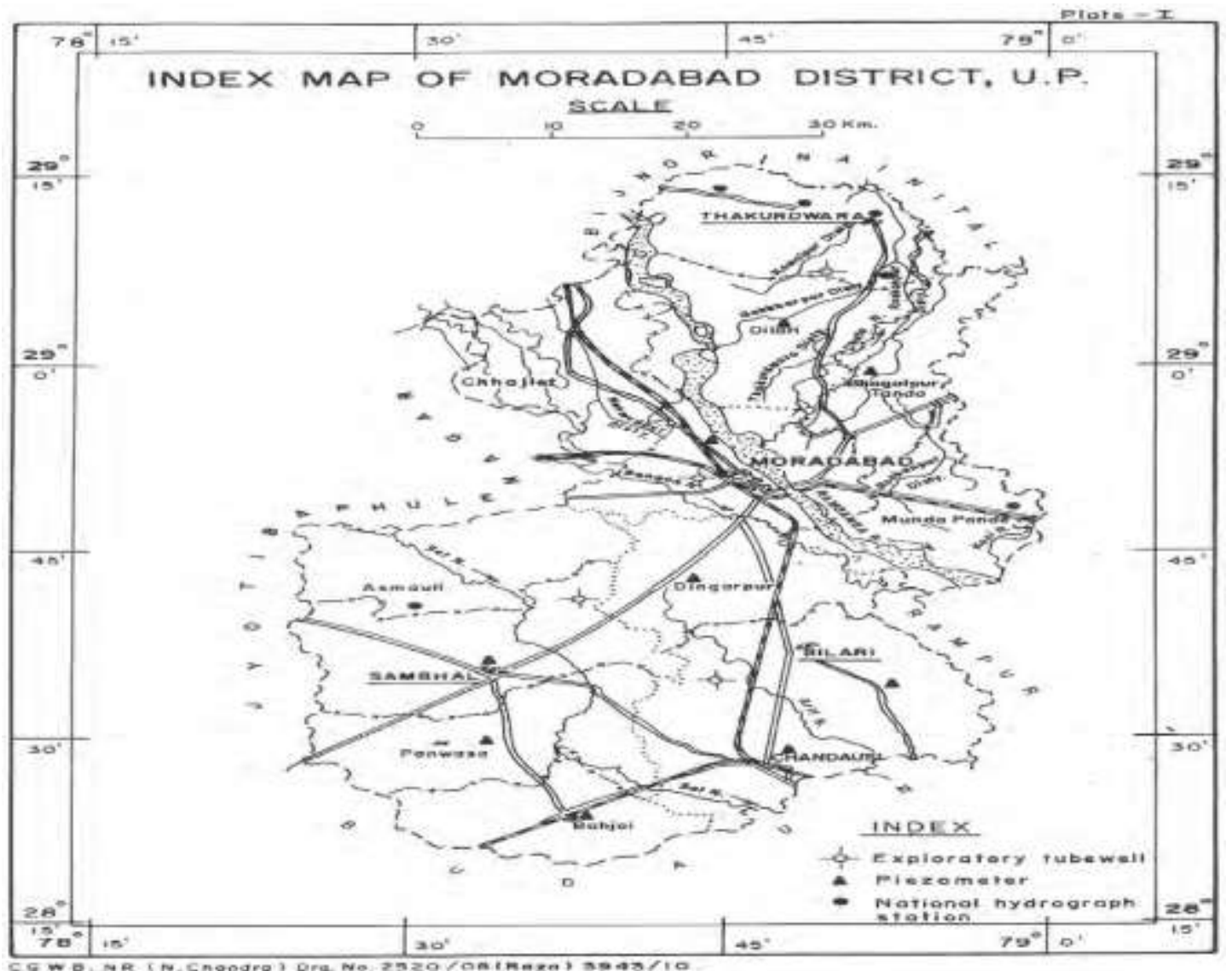

Trace Metal concentration of densely populated area of Moradabad city

\begin{tabular}{|l|l|l|l|l|l|l|l|l|l|l|l|l|l|}
\hline \multicolumn{10}{|c|}{ Trace Metal concentration of densely populated area of Moradabad city } \\
\hline MandiSamiti Site-1 & \multicolumn{10}{|c|}{ Locoshedbridge Site -2 } \\
\hline Parameter & Summer & Rainy & Winter & SD \pm & Average & NPI & Summer & Rainy & Winter & SD \pm & Average & NPI \\
\hline Fe & 0.409 & 0.398 & 0.51 & 0.0617 & 0.439 & 1.46 & 0.544 & 0.614 & 0.46 & 0.0771 & 0.54 & 1.8 \\
\hline $\mathbf{P b}$ & 0 & 0.024 & 0 & 0.0138 & 0.008 & 0.16 & 0 & 0.029 & 0.33 & 0.1827 & 0.12 & 2.4 \\
\hline $\mathbf{C r}$ & 0.03 & 0.02 & 0.01 & 0.01 & 0.02 & 0.4 & 0.03 & 0.02 & 0.01 & 0.01 & 0.02 & 0.4 \\
\hline $\mathbf{N i}$ & 0.01 & 0.01 & 0.01 & 0 & 0.01 & 0.2 & 0.01 & 0.011 & 0 & 0.006 & 0.007 & 0.14 \\
\hline $\mathbf{Z n}$ & 1.178 & 1.96 & 0 & 0.9866 & 1.046 & 0.21 & 2.622 & 0.204 & 1.173 & 1.2169 & 1.333 & 0.27 \\
\hline $\mathbf{C u}$ & 0.02 & 0.01 & 0.02 & 0.0057 & 0.0166 & 0.332 & 0.02 & 0.024 & 0.061 & 0.0226 & 0.035 & 0.7 \\
\hline
\end{tabular}

\begin{tabular}{|l|l|l|l|l|l|l|l|l|l|l|l|l|}
\hline MDA Site -3 & \multicolumn{1}{l|}{ Civil Lines Site -4 } \\
\hline Parameter & Summer & Rainy & Winter & SD \pm & Average & NPI & Summer & Rainy & Winter & SD \pm & Average & NPI \\
\hline Fe & 0.919 & 0.643 & 0.31 & 0.3049 & 0.624 & 2.08 & 0.432 & 0.425 & 0.12 & 0.1781 & 0.326 & 1.08 \\
\hline Pb & 0 & 0.031 & 0.026 & 0.0166 & 0.019 & 0.38 & 0.013 & 0 & 0.028 & 0.014 & 0.0136 & 0.27 \\
\hline $\mathbf{C r}$ & 0.03 & 0.01 & 0.03 & 0.0115 & 0.023 & 0.46 & 0.03 & 0 & 0.02 & 0.0152 & 0.0166 & 0.33 \\
\hline $\mathbf{N i}$ & 0.01 & 0.022 & 0.01 & 0.0069 & 0.014 & 0.28 & 0.03 & 0.018 & 0.01 & 0.01 & 0.0193 & 0.38 \\
\hline $\mathbf{Z n}$ & 0.452 & 0.755 & 0.38 & 0.199 & 0.529 & 0.11 & 0.199 & 0.699 & 0 & 0.3601 & 0.299 & 0.06 \\
\hline $\mathbf{C u}$ & 0.01 & 0.026 & 0.04 & 0.015 & 0.025 & 0.5 & 0.01 & 0.036 & 0.002 & 0.0177 & 0.016 & 0.32 \\
\hline
\end{tabular}

\begin{tabular}{|l|l|l|l|l|l|l|l|l|l|l|l|l|}
\hline Moradabad Club Site-5 & \multicolumn{10}{l|}{ Harthala Colony Site -6 } \\
\hline Parameter & $\begin{array}{l}\text { Summe } \\
\mathbf{r}\end{array}$ & $\begin{array}{l}\text { Rain } \\
\mathbf{y}\end{array}$ & $\begin{array}{l}\text { Winte } \\
\mathbf{r}\end{array}$ & $\mathbf{S D} \pm$ & $\begin{array}{l}\text { Averag } \\
\mathbf{e}\end{array}$ & $\mathbf{N P I}$ & Summer & Rainy & $\begin{array}{l}\text { Winte } \\
\mathbf{r}\end{array}$ & $\mathbf{S D} \pm$ & $\begin{array}{l}\text { Averag } \\
\mathbf{e}\end{array}$ & NPI \\
\hline $\mathbf{F e}$ & 0.484 & 0.28 & 0.34 & 0.104 & 0.368 & 1.23 & 0.612 & 0.779 & 0.62 & 0.0941 & 0.67 & 2.23 \\
\hline $\mathbf{P b}$ & 0.024 & 0 & 0.009 & 0.0121 & 0.011 & 0.22 & 0.047 & nt & 0.034 & 0.0091 & 0.027 & 0.54 \\
\hline $\mathbf{C r}$ & 0.07 & 0.01 & 0.02 & 0.0321 & 0.033 & 0.66 & 0.01 & 0.04 & 0.01 & 0.0173 & 0.02 & 0.04 \\
\hline $\mathbf{N i}$ & 0 & 0 & 0 & 0 & ---- & ---- & 0.01 & 0.026 & $\mathrm{nt}$ & 0.0113 & 0.012 & 0.24 \\
\hline $\mathbf{Z n}$ & 0.467 & 0 & 0 & 0.2696 & 0.156 & 0.03 & 0.944 & 0.867 & $\mathrm{nt}$ & 0.0544 & 0.604 & 0.12 \\
\hline $\mathbf{C u}$ & 0.02 & 0.039 & 0.007 & 0.016 & 0.022 & 0.44 & 0.01 & 0.03 & $\mathrm{nt}$ & 0.0141 & 0.0133 & 0.27 \\
\hline
\end{tabular}

\begin{tabular}{|l|l|l|l|l|l|l|l|l|l|l|l|l|}
\hline \multicolumn{1}{|l|}{ Mahila Thana Site -7 } \\
\hline $\begin{array}{l}\text { Paramete } \\
\mathbf{r}\end{array}$ & $\begin{array}{l}\text { Summe } \\
\mathbf{r}\end{array}$ & $\begin{array}{l}\text { Rain } \\
\mathbf{y}\end{array}$ & $\begin{array}{l}\text { Winte } \\
\mathbf{r}\end{array}$ & $\mathbf{S D} \pm$ & $\begin{array}{l}\text { Averag } \\
\mathbf{e}\end{array}$ & $\mathbf{N P I}$ & $\mathbf{S u m m e r}$ & $\mathbf{R a i n y}$ & $\begin{array}{l}\text { Winte } \\
\mathbf{r}\end{array}$ & $\mathbf{S D} \pm$ & $\begin{array}{l}\text { Averag } \\
\mathbf{e}\end{array}$ & NPI \\
\hline $\mathbf{F e}$ & 2.08 & 0.872 & 0.847 & 0.7047 & 0.15 & 0.623 & 0.958 & 0.666 & 0.031 & 0.4739 & 0.55 & 1.84 \\
\hline $\mathbf{P b}$ & 0.39 & 0.018 & 0.011 & 0.2168 & 0.029 & 0.019 & 0 & 0 & 0.034 & 0.0196 & 0.0113 & 0.23 \\
\hline $\mathbf{C r}$ & 0.27 & 0.02 & 0.01 & 0.1473 & 0.01 & 0.013 & 0.03 & 0.02 & 0.02 & 0.0057 & 0.023 & 0.47 \\
\hline $\mathbf{N i}$ & 0.066 & 0.01 & 0 & 0.0355 & 0 & 0.003 & 0 & 0.019 & 0.01 & 0.0095 & 0.0097 & 0.19 \\
\hline $\mathbf{Z n}$ & 0.095 & 0.186 & 0.867 & 0.4219 & 0 & 0.475 & 3.848 & 2.004 & 0 & 1.9245 & 1.95 & 0.39 \\
\hline
\end{tabular}


Indexing of Under Groundwater Quality in Urban Area of Moradabad District in Northern India

\begin{tabular}{|c|c|c|c|c|c|c|c|c|c|c|c|c|}
\hline $\mathbf{C u}$ & 0.05 & 0.01 & 0.03 & 0.02 & 0.007 & 0.016 & 0.03 & 0.01 & 0.035 & 0.0132 & 0.025 & 0.5 \\
\hline \multicolumn{7}{|c|}{ Jain Mandir Site -9 } & \multicolumn{6}{|c|}{ Harpal Nagar Site -10 } \\
\hline $\begin{array}{l}\text { Paramete } \\
\mathbf{r}\end{array}$ & $\begin{array}{l}\text { Summe } \\
\mathbf{r}\end{array}$ & $\begin{array}{l}\text { Rain } \\
\mathbf{y}\end{array}$ & $\begin{array}{l}\text { Winte } \\
\mathbf{r}\end{array}$ & SD \pm & $\begin{array}{l}\text { Averag } \\
\text { e }\end{array}$ & NPI & Summer & Rainy & $\begin{array}{l}\text { Winte } \\
\mathbf{r}\end{array}$ & SD \pm & $\begin{array}{l}\text { Averag } \\
\text { e }\end{array}$ & NPI \\
\hline $\mathbf{F e}$ & 0.642 & 0.636 & 0.032 & 0.3504 & 0.436 & 1.45 & 0.499 & 0.59 & 0.45 & 0.071 & 0.513 & 1.71 \\
\hline $\mathbf{P b}$ & 0 & 0 & 0.03 & 0.0173 & 0.01 & 0.2 & 0.016 & 0.026 & 0.038 & 0.011 & 0.026 & 0.53 \\
\hline $\mathbf{C r}$ & 0.02 & 0 & 0 & 0.0115 & 0.0066 & 0.133 & 0.01 & 0.02 & 0.02 & 0.0057 & 0.016 & 0.33 \\
\hline $\mathbf{N i}$ & 0 & 0 & 0.01 & 0.0057 & 0.0033 & 0.066 & 0.01 & 0 & 0.04 & 0.0208 & 0.0166 & 0.33 \\
\hline $\mathbf{Z n}$ & 2.717 & 0 & 0 & 1.5686 & 0.906 & 0.18 & 2.385 & 1.296 & 0 & 1.1939 & 1.227 & 0.25 \\
\hline $\mathrm{Cu}$ & 0.02 & 0.056 & 0.051 & 0.0195 & 0.042 & 0.85 & 0.09 & 0 & 0.006 & 0.0503 & 0.032 & 0.64 \\
\hline
\end{tabular}

TABLE 2: Various samples point location to serial number in less densely populated area of Moradabad city

\begin{tabular}{|l|l|l|l|}
\hline S.No. & Location & Source & Depth (In Feet) \\
\hline 1 & MandiSamiti Site-1 & Hand Pump & 40 \\
\hline 2 & Locoshedbridge Site -2 & Hand Pump (Indian Mark - II) & 120 \\
\hline 3 & MDA Site -3 & Hand Pump (Indian Mark - II) & 120 \\
\hline 4 & Civil Lines Site -4 & Hand Pump & 45 \\
\hline 5 & Moradabad Club Site-5 & Hand Pump (Indian Mark - II) & 120 \\
\hline 6 & Harthala Colony Site -6 & Hand Pump & 35 \\
\hline 7 & Mahila Thana Site -7 & Hand Pump & 40 \\
\hline 8 & DyptiGanj Site -8 & Hand Pump & 40 \\
\hline 9 & Jain Mandir Site -9 & Hand Pump & 40 \\
\hline 10 & Harpal Nagar Site -10 & Hand Pump (Indian Mark - II) & 120 \\
\hline
\end{tabular}

All values in $\mathrm{mg} / \mathrm{L}$ except $\mathrm{pH}$ and turbidity (in NTU) $\mathrm{S}=$ Summer $\mathrm{R}=$ Rainy $\mathrm{W}=$ winter WHO = World Health Organization CPHEEO = Central Public Health and Environmental Engineering Organization

\section{Conclusion}

Groundwater is the main source of potable water of Moradabad City. A number of people have installed water purifier in their houses in order to get rid of any contamination in water. The main source of groundwater contamination is from domestic and a number of Electroplating, pulp and paper,dairy and sugar industries etc.

These industries pour their effluents directly into open drain, unlined earthen drain and openpits in several places from where polluted water percolates into soil and finally reaches theaquifers. The data collected led to the conclusion that concentration of indicative parameters in ground water are site specific and are highly variable. A water quality index give effects of various parameters. To sum up the findings the sub surface water of the experimental Area of Moradabad is moderately fit for drinking purposes. However, parameters varied even from sample to sample. The NPI index of most of the parameters in ground water quality did not exceed limits of 1.00 with a few exceptions. The seasons also influenced the ground water quality of the region. It seems that on precautionary basis, the measures could be taken at this stage before the pollution status deepens in the outskirts of the Moradabad city in coming times and passes the stage of irreparable rectification.

\section{References}

[1]. APHA 1992.Standard methods for the examination of water and waste. American Public Health Association, New York, USA.

[2]. Maduri Usha T., Srinivas, T. and Sireesha, K. 2004. Pollution Res., 23(2): 565-568.

[3]. Mohan Anuraag., Singh.R.K, Pandey Kirti., Kumar Vineet., Jain.V.,2007.IJEP 27( 11):1031-1035.

[4]. Singh Rajeev Kumar, Mohan Anuraag., Jain.,V.2016,IOSR-JAC 9,(4 Ver,I):32-36

[5]. WHO 1993.International standards for drinking water. World Health Organization, Geneva

Authors:

[6]. Dr. *Rajeev Kumar Singh, Assistant professor, SRMSCET,BAREILLY (919411004262)Email, katheria.rajeev@ gmail.com

[7]. Dr. Ritu Singh,Assistant professor, SRMSCETR,BAREILLY:Email, singh.ritu1001@ gmail.com 